\title{
Rheological behavior of sewage sludge and strain-induced dewatering
}

\author{
Fami Chaari \\ Guillaume Racineux \\ Arnaud Poitou \\ Mohend Chaouche
}

\begin{abstract}
In order to study the strain-induced water release in sewage sludge and its connection with rheological behavior, two types of rheological tests have been carried out. The rheology of sewage sludge samples stemming from a urban sewer was first characterized using a Couette cell system. In particular, the yielding behavior and the elastic modulus of the sludge has been considered under shear flow conditions. In these pure shear tests the reproducibility of the measurements was rather poor, limiting this study to low strains. Consequently, a second type of rheological tests, namely the squeeze test, which is more
\end{abstract}

appropriate for these paste-like materials, has been considered. The rheological behavior along with the dewatering efficiency have been studied under the squeeze flow conditions. Surprisingly, it was found that, under certain conditions, the strain-induced water release mechanism became more effective when decreasing the squeeze speed. This was interpreted in terms of a competition between the paste flow and the water filtration through the porous media made up by the flocs.

Keywords Rheology - Sludge · Squeeze flow · Yield Stress ·

Filtration

\section{Introduction}

Removing the maximum possible amount of water from sewage sludge is a great challenge, both industrially and environmentally. In general, typical industrial sludge processing consists of three main steps: physico-chemical treatment (flocculation and then decantation), mechanical processing (centrifugation, compression, etc.), and finally the residual product is either used as a fertilizer in agriculture or incinerated. We are interested here in the second step of the process, namely the effect of mechanical solicitations on the water-flocs separation.

Due to the high complexity of urban sewage sludge composition, we do not intend here to undertake a quantitative analysis, relating the mechanical behavior to the microstructure dynamics. A number of more quantitative studies can be found in the literature concerning the rheological behavior of model materials, which are assumed to be representative of sewage sludges (Kao et al. 1998; Legrand 1997). Since in our study we deal with a 'real' urban sludge, only a qualitative analysis can be undertaken. This mainly consists of bringing out some of general trends of its rheological behavior and comparing them to other paste-like materials.

Some studies of the rheological behavior of urban sewage sludges are reported in the literature; however most of them deal with the sludge in the 'liquid' state. Thus, sludge in this state was found to be in general thixotropic (Campbell and Crescuollo 1982; Honey and Pretorius 2000), and was characterized using different rheological models, including the Bingham (Lotito et al. 1997; Chilton et al. 1995) and the Herschel-Bulkley models (Baudez 2001; Mulbarger et al. 1981). These rheological studies were conducted to understand the flow properties of the sewage sludge, in order to optimize their transport processes. 
In our case, the sludge is subjected to a physicochemical treatment prior to the rheological tests, giving rise to a paste-like material (highly concentrated suspension). Rheological studies of such materials are rather rare. Recently, Baudez and Coussot (Baudez 2001; Baudez and Coussot 2001) reported a study of the rheological behavior of a similar material. They considered the long time rheological behavior of the sludge, and their study is complimentary to ours.

The objective of the present study is twofold. First, we consider the shear-rheological behavior of sludges whose solid content is higher than in the studies we referred to above. In our case the sludge is in a gel state. In particular, the yielding and elastic properties of the sludge are considered. Second, squeeze tests are conducted in order to investigate the compression-induced dewatering. Surprisingly, it is found that under certain conditions the squeeze-induced dewatering becomes more effective at small squeeze velocities. This may have important technological consequences. Our results are interpreted by assuming a competition between the sludge deformation as a whole and the water filtration through the porous media made-up by the flocs. To our knowledge, this is the first time such a study is reported with sludge. It is to be noted that a similar squeeze behavior has already been reported, but with quite different materials, including a model suspension of spheres (Delaye et al. 2000) and ceramic pastes (Poitou and Racineux 2001).

\section{Experiments}

Materials The sludge samples used in our experiments stemmed from the urban sewer of Valenton (France), and was kindly supplied by RHODIA-France. In order to freeze its biological activity, and consequently its microstructure evolution with time due to a too complex process, which we would like to avoid here, the sludge has been divided into several small samples and stocked in a fridge. Moreover, the sludge has actually been subjected to a chemical processing by RHODIA in order to stop its biological activity.

Similar to the common industrial procedures, the sludge samples have been subjected to a physico-chemical treatment prior to mechanical tests. That is, a flocculent was added to the sewer sample. After a certain time period (which was the same for all the samples) of the flocculation and decantation process, the released water was removed. The remaining sludge, which was a gel-like paste material, was then loaded into the rheological cell and mechanical tests were undertaken.

Shear-rheological behavior The rheological behavior of the sludge paste was first characterized using a shear rheometer apparatus. The latter was a Stresstech (from Rheologica) which can detect a torque as small as $10^{-8} \mathrm{~N} \mathrm{~m}$, and the position resolution of the sensor is as accurate as $1 \mu \mathrm{rd}$. This enabled us to carry out accurate measurements even at quite low shear stresses. All the measurements were made in a Couette geometry (concentric cylinders) in order to produce uniform shear deformations at the length scale of the floc size, which was less than $0.1 \mathrm{~mm}$. The diameter of the inner cylinder was $50 \mathrm{~mm}$ and the gap between the cylinders was $1 \mathrm{~mm}$, corresponding to several floc sizes. The Couette cell geometry was also chosen because the sedimentation effects on the rheological measurements may be smaller compared to the other common rheological cells. However, sedimentation effects would have not been significant due the high viscosity of the paste.

Different rheological properties of the sludge have been investigated. However, due to the very likely occurrence of fracture and wall slippage, we mainly focus here on the small deformation properties of the material, including the elastic modulus and the yielding properties. The rheological behavior of a similar material at large strains has recently been reported by Baudez and Coussot (2001). They showed in particular that we actually have the problem of wall-slip and fracture with such material.

Sewage sludges have often been reported as thixotropic materials. This rheological aspect has been tentatively investigated. However, it was found that thixotropy, which mainly refers to reversible effects, cannot be properly used to characterize the sludge used here. Under shear, the material was suspected to undergo rather irreversible deflocculation. This is in line with the results of Baudez and Coussot (2001).

Yielding properties It is well-known that there is some controversy in the literature concerning the definition of material yield stress (Barnes 1999). In the present study, the measurement method adopted is the following. The sample is subjected to an increasing controlled stress and the resulting shear rate is measured. For a material with a yielding behavior there is a dramatic increase of the slope of the curve representing the shear rate as a function of the stress above a certain critical stress. The latter is defined to be the yield stress of the material. Such a behavior is actually observed in the case of our material. The experimental results are represented in Fig. 1.

The yield stress of the sample whose behavior is represented in Fig. 1 is about $250 \mathrm{~Pa}$.

The physical origin of the yield stress of the sludge paste can be roughly attributed to the required hydrodynamic drag force to break the largest flocs in the system, more specifically, the flocs linking up two opposite boundaries of the shearing cell. The hydrodynamic force necessary to break a link between particles making-up a floc can be estimated as $\mathrm{F}_{\mathrm{h}} \sim 6 \pi \mathrm{R}^{2} \sigma_{\mathrm{y}}$, where $\mathrm{R}$ is the particle radius and $\sigma_{\mathrm{y}}$ is the yield stress. For a given applied stress $\left(\sigma_{\mathrm{y}}\right)$, the hydrodynamic

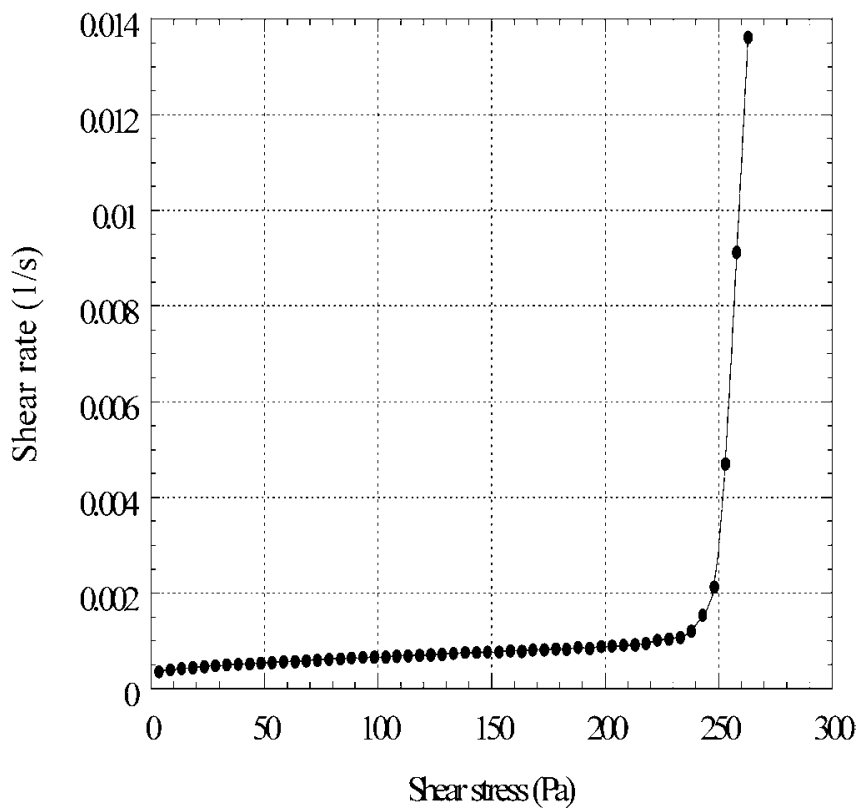

Fig. 1 Evolution of the shear rate as a function of shear stress. The yield stress $\sigma_{\mathrm{y}}$ is the stress for which there is a significant increase of the shear rate. In this case $\sigma_{\mathrm{y}} \approx 250 \mathrm{~Pa}$ 
force is maximum for the largest particles, whose size is about $0.1 \mathrm{~mm}$ for the sludge used in our experiments. $F_{h}$ is then about $10^{-5} \mathrm{~N}$, which is much larger than the forces with molecular origin, such as the van der Waals forces (about $10^{-9} \mathrm{~N}$ ). This suggests that the yield stress measured here may be actually related to a macroscopic force, such as mechanical friction among the particles and between the particles and the cell walls. We did not investigate in our study the physical origin of our material yield stress.

The yielding properties of the sludge can also be investigated by considering its elastic properties at low strains. This is reported in the next subsection.

Elastic properties Below the yield stress the material would be elastic. In order to investigate this elastic domain, the material is subjected to small strains at different shear rates, and the corresponding stresses are measured. Such tests are very common when considering mechanical properties of solid materials, but not with fluids.

The evolution of the stress as a function of the strain is represented in Fig. 2 for different shear rates. Below a certain strain, the stress-strain relationship is almost independent of the shear rate, except for very low shear rates. The strain for which the material starts to 'flow' corresponds to a stress of about $300 \mathrm{~Pa}$, which is consistent with the previous measurements of the yield stress. If we assume that the material is elastic, we can measure the elastic modulus. However, contrary to a purely elastic material, the elastic modulus (slope of the curve stress-strain) depends, although slightly, on the deformation rate. This suggests that, even below the yield stress as defined here, there may be some links' breakage in the largest flocs. This would also explain why the estimated hydrodynamic drag force necessary to induce a significant flow is so large compared to the molecular forces.

For large strains (flow regime), the experimental results are poorly reproducible, and are not reported here. One of the reasons would be the formation of low-viscosity shear bands and other mechanical instabilities. We did not investigate further the shear flow behavior of the sludge. Such study using a similar material have been recently undertaken by Baudez and Coussot (2001).

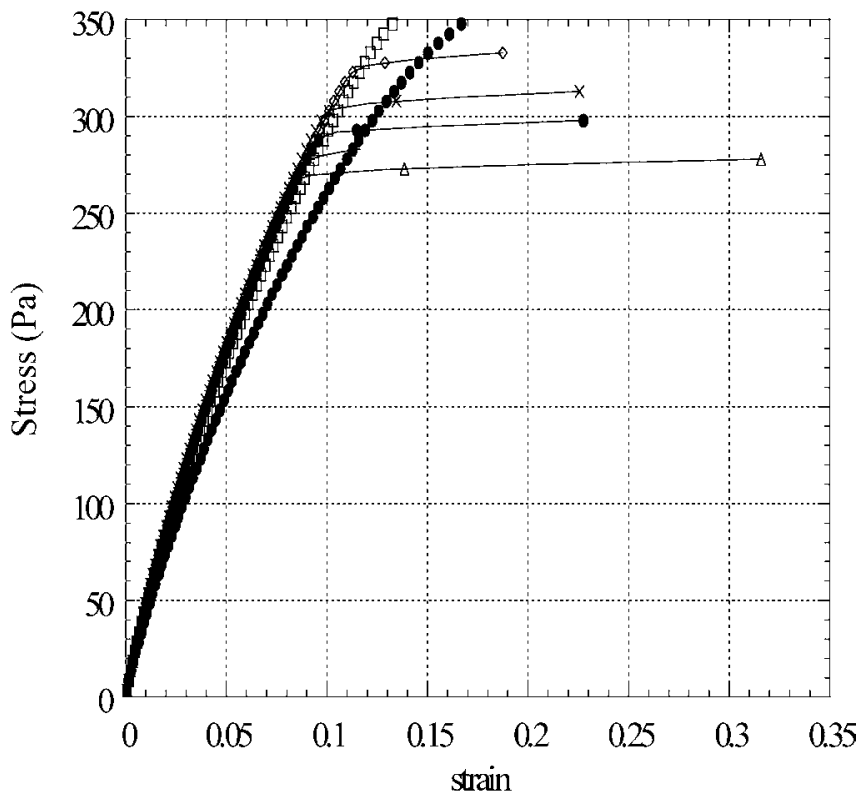

Fig. 2 Representation of the stress as a function of the strain for different shear rates, making evidence of the elastic behavior of the material at small strains
Since this material is rather a paste-like material, another, more appropriate, deformation cell geometry (squeeze cell) is used. This geometry has been proven to be much more appropriate in studying pastes (Poitou and Racineux 2001; Ravilly 2000; Adams et al. 1997) and highly concentrated suspensions (Delaye et al. 2000).

Squeeze tests - experimental set up

The sludge samples are squeezed out between two parallel discs mounted on a compression-traction machine (from MTS). The lower disc can be displaced at controlled velocities, while the upper one is maintained stationary. The latter experiences a normal force which is recorded as a function of time for each fixed velocity. In all the experiments, the initial disc separation $\mathrm{h}_{0}$ is taken to be $4 \mathrm{~mm}$, which is much smaller than the disc diameter $(27 \mathrm{~mm})$. In order to minimize wall-slippage of the sample, the inner surface of the discs are coated with thin sand papers. Since the traction-compression machine used here is designed for studying rather metal materials, the uncertainty in the force measurements is quite high $( \pm 2 \mathrm{~N})$. On the other hand, the disc separation (velocity) is measured with a much higher accuracy, namely $\pm 1 \mu \mathrm{m}$.

\section{Rheological results and discussions}

The evolution of the normal force $\mathrm{F}$ as a function of normalized disc separation $\left(\mathrm{h} / \mathrm{h}_{0}\right)$ is represented in Fig. 3 for a separation speed range which nearly spans four orders of magnitude $(0.05 \mathrm{~mm} / \mathrm{s} \rightarrow 100 \mathrm{~mm} / \mathrm{s})$. Examining Fig. 3, the following remarks can be drawn:

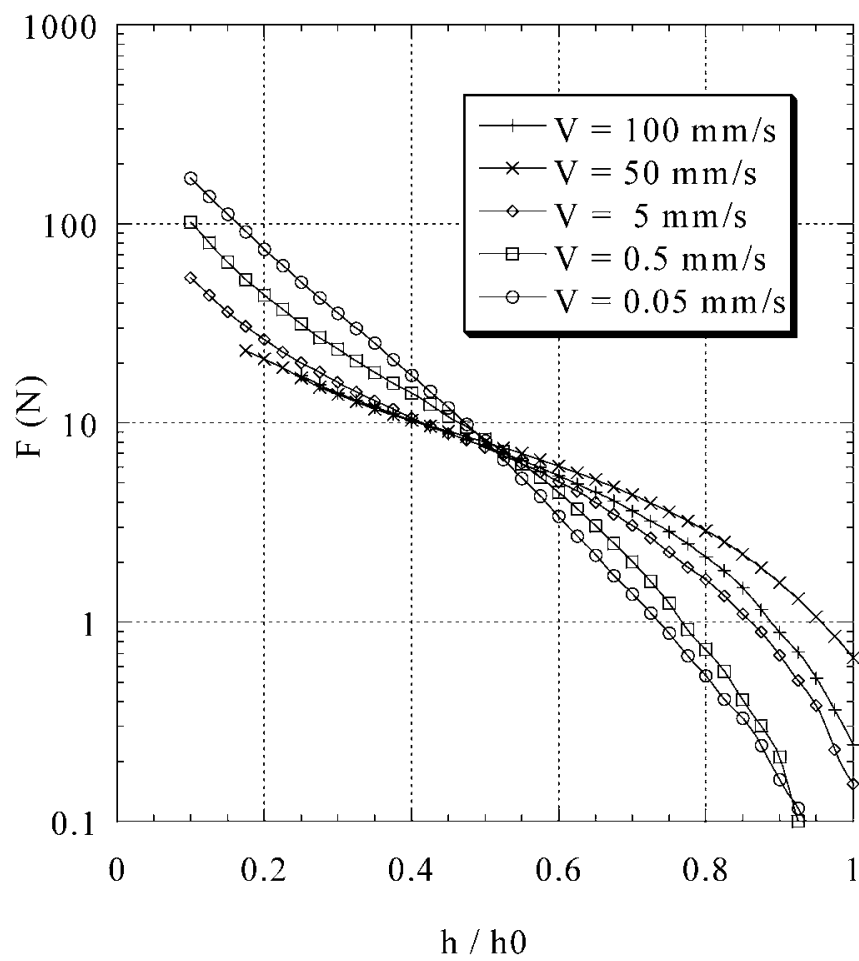

Fig. 3 Squeezing force vs normalized disc separation 
1. When the normalized disc separation is larger than 0.5 , the squeezing force is an increasing function of the disc speed for any level of disc separation. This behavior is expected and is consistent with that of a viscous, and here non-Newtonian, fluid.

2. When the normalized disc separation is smaller that 0.5 , two deformation regimes can be distinguished. For small disc speeds $(5 \mathrm{~mm} / \mathrm{s}, 0.5 \mathrm{~mm} / \mathrm{s}, 0.05 \mathrm{~mm} /$ $\mathrm{s})$, the squeezing force increases when the velocity decreases, while for large speeds $(50 \mathrm{~mm} / \mathrm{s}$, $100 \mathrm{~mm} / \mathrm{s}$ ), the force seems to be independent of the speed.

The squeeze flow behavior described above is similar to other paste-like materials as already reported in the literature (Delaye et al. 2000; Poitou and Racineux 2001). The fact that the squeezing force may increase when disc speed decreases can be attributed to the water filtration through the porous media made up by the flocs, which would result in a solid concentration increase of the sample during the squeeze test (Fig. 4). This is discussed below in more details. For high speeds, the squeeze force is found to be independent on the speed. The material has then a plastic behavior.

The most significant parameters that control the transition reported in the above experiments can be inferred by assuming a diphasic behavior of the sludge paste. A model based on such an assumption has been successfully used to describe the rheological behavior of ceramic pastes (Lanteri et al. 1996; Poitou and Racineux

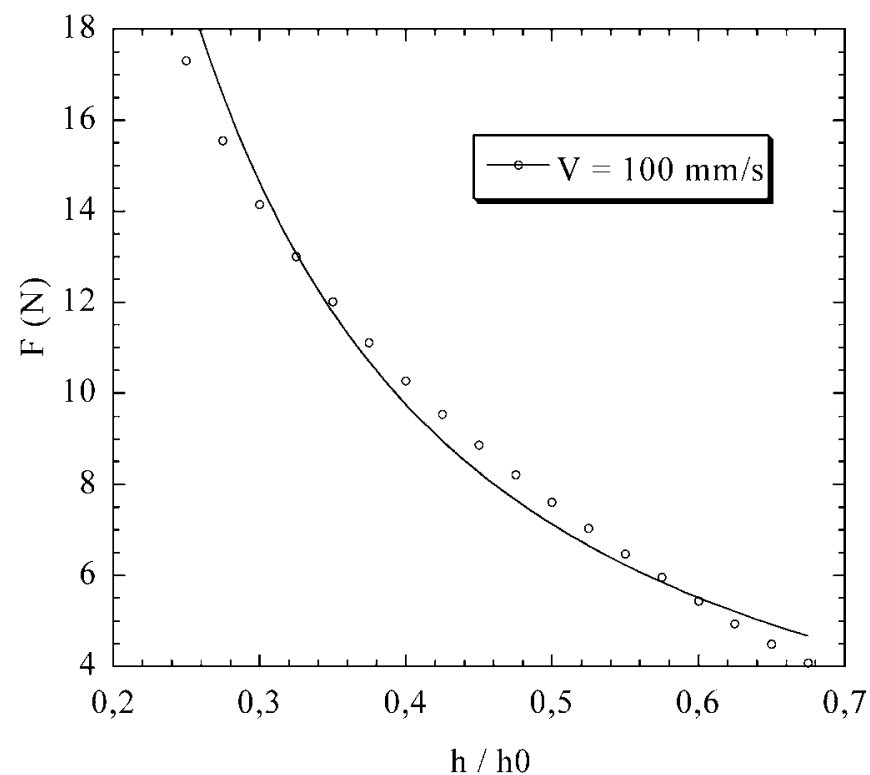

Fig. 4 Representation of the best fit between the theoretical prediction (continuous curve) of the squeeze force and the measurements in the case of a 'homogeneous' deformation of the sludge sample. The best fit gives the values of the shear-thinning index and the effective viscosity of the material
2001) and model suspensions (Delaye et al. 2000). In this approach, the material is assumed to deform with two different flow fields $v_{p}$ and $v_{f}$, associated, respectively, with the particles and the fluid. This vision is different from what is generally assumed for flow of suspensions, in which the particles are supposed to move at the same velocity as the fluid. Numerical simulations based on such a model has been used by Racineux (1999) to describe quantitatively the squeeze deformation behavior of ceramic pastes. In this discussion we just give a qualitative analysis comparing the time scales of the two phenomena taking place when squeezing a paste-like material.

We assume that the sample sludge can be pictured as a deformable porous media made up by the flocs. The water flow relative to the flocs can be described by the Darcy's law, which can be written as

$v_{f}-v_{s}=-\frac{k(\phi)}{\eta_{w}} \operatorname{grad} p$

where $\eta_{w}$ is the water viscosity, grad $\mathrm{p}$ the pressure gradient giving rise to the water flow, and $\mathrm{k}(\phi)$ the Darcy's permeability, which scales with the mean floc size squared. $\mathrm{k}(\phi)$ is also a function of the solid volume fraction $\phi$, although this dependence is rather weak. For instance, if we assume that the permeability obeys the Kozeny-Carman model (Carman 1937), we have $\mathrm{k}(\phi)$ $\propto(1-\phi)^{3} / \phi^{2}$.

The deformation of the porous media can be assumed to correspond to the flow of a power-low fluid, which is quite general for paste-like materials. Thus, the stress related to this deformation can be written as

$\sigma=A(\phi) \dot{\gamma}^{m} \propto A(\phi)\left(\frac{U}{h}\right)^{m}$

where $\dot{\gamma}$ is the average shear rate, and $\mathrm{m}$ the shearthinning (if $m<1$ ) or shear thickening (if $m>1$ ) index of the paste. $\mathrm{A}(\phi)$ is the consistency (or effective viscosity) of the paste, which is a strong function of the concentration, in particular when the latter is close to its 'jamming' value. The effective viscosity of a material, made up by a concentrated collection of solid particles in a viscous fluid, actually diverges when the solid volume fraction approaches its maximum value. A number of experimental, numerical and phenomenological approaches lead to a power-law divergence (Russel et al. 1989), that is $\mathrm{A}(\phi) \sim \mu_{\mathrm{w}}(1-\phi / \phi \max )^{-\alpha}$, where the exponent $\alpha$ is a function of the particle's geometrical form and the type of interactions the particles exert on each other.

As is shown in the previous section, the sludge is a yield stress fluid. The value of the yield stress $\sigma_{\mathrm{y}}$ was found to be about $250 \mathrm{~Pa}$. One has then to take this into account in Eq. (2). However, the yield force $F_{y}$ $\left(\mathrm{F}_{\mathrm{y}}=\pi \mathrm{R}^{2} \sigma_{\mathrm{y}}=0.14 \mathrm{~N}\right.$, where $\mathrm{R}$ is the disc radius) is much 
smaller than the forces involved in the squeeze experiments (see Fig. 3). We then neglect the yield stress in this discussion and consider that the material is a power-law fluid.

In mechanical equilibrium, we have div $\sigma$-grad $\mathrm{p}=0$. Under the Hele-Shaw approximation conditions (disc separation much smaller than the disc radius), one has

$\operatorname{grad} \mathrm{p} \propto \mathrm{s} / \mathrm{h} \propto \mathrm{A}(\phi)(\mathrm{U} / \mathrm{h})^{\mathrm{m}} / \mathrm{h}$.

Then, using the Darcy's law, we can estimate the time scale for the water filtration through the media, which can be defined to be the time necessary for the water to flow over a distance equal to the disc separation, that is

$\tau_{w} \propto \frac{\eta_{w} h^{2}}{A(\phi) k(\phi)}\left(\frac{h}{U}\right)^{m}$

The time scale of the paste deformation is roughly: $\tau_{\mathrm{s}} \propto 1 / \propto \mathrm{h} / \mathrm{U}$.

The filtration phenomenon yields a concentration increase which in turn increases the force necessary to squeeze out the sample, due the strong dependence of the paste consistency upon the concentration. This phenomenon becomes significant when the two time scales estimated above are comparable. Using the Darcy's law along with the conservation law of the liquid phase, one can show that the fluid filtration is a diffusion-like phenomenon. On the other hand, the paste flow obeys a convection equation. Then a Peclet number can be defined using the two time scales, that is

$P e=\frac{\tau_{s}}{\tau_{w}}=\frac{A(\phi) k(\phi) U^{m-1}}{\eta_{w} h^{m+1}}$

For Pe $\gg 1$, the filtration mechanism is expected to be significant and the squeeze force would increase when the velocity decreases. If the material is shear-thinning $(\mathrm{m}<1)$ this would happen for small speeds, while for shear-thickening materials it would take place for large speeds. We will see below that our material is actually highly shear-thinning. The velocity dependence of the Peclet number is then consistent with our experimental findings.

For $\mathrm{Pe} \ll 1$, the water filtration is expected to be negligible, and the material would remain homogeneous during the squeezing process. This would happen for large speeds for shear-thinning materials.

For the singular case of a Newtonian paste flow, which corresponds to $\mathrm{m}=1$ and is not actually realistic for such materials, the Peclet number is expected to be independent of the squeeze speed.

The 'critical' speed separating the two squeeze rheological behaviors found in our experiments can be assumed to correspond to $\mathrm{Pe}=0(1)$, which leads to
$U_{c}=\left(\frac{A(\phi) k(\phi)}{\eta_{w} h^{m+1}}\right)^{1 / 1-m}$

It is worth noting that this result can be recovered using energetic considerations (Poitou and Racineux 2001).

The power law index and the consistency of the medium can be measured experimentally under the conditions for which the filtration mechanism is negligible, that is for high speeds. In the case of our material, according the rheological behavior, we can consider that for a squeeze speed of $100 \mathrm{~mm} / \mathrm{s}$ the water filtration is negligible. If we assume that the material deforms as a power-law fluid, it is straightforward to show that, under the Hele-Shaw approximation conditions, the expression of the squeeze force is (Scott 1935)

$F=2 \pi\left(\frac{2 m+1}{m}\right)^{m} \frac{A}{m+3} \frac{U^{m}}{h^{2 m+1}} R^{m+3}$

Figure 5 represents the best fit of the above expression of the squeeze force with our experimental results for a speed of $100 \mathrm{~mm} / \mathrm{s}$. The shear-thinning index and the consistency of the material can then be inferred. The best fit gives $\mathrm{m}=0.2$ and $\mathrm{A}=598 \mathrm{~Pa} \mathrm{~s}^{\mathrm{m}}$. Our material is thus highly shear-thinning and 'viscous'.

The critical velocity for which we found a significant change of the rheological behavior of the sludge due to the water filtration mechanism can be estimated to be between $50 \mathrm{~mm} / \mathrm{s}$ and $100 \mathrm{~mm} / \mathrm{s}$. Using these values of the speed along with the experimental values of the

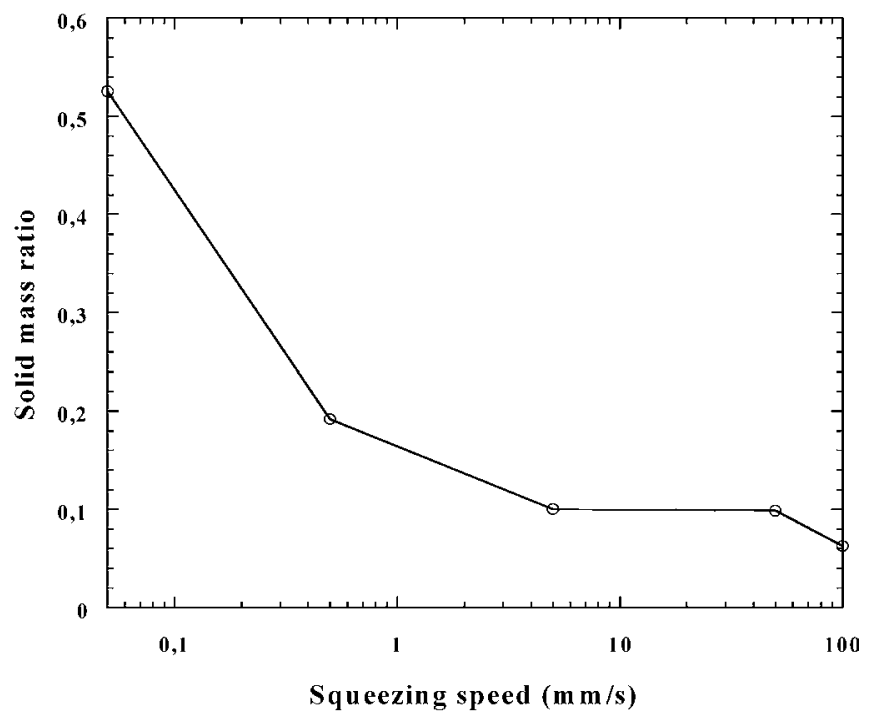

Fig. 5 Evolution of the dewatering efficiency as a function of the squeeze speed 
parameters $\mathrm{m}$ and $\mathrm{A}$, and taking $\mathrm{h}=2 \mathrm{~mm}$ in the derived expression of the Peclet number, we find that $\mathrm{Pe}=1$ would be consistent with a Darcy' permeability bounded by $0.9 \times 10^{-10} \mathrm{~m}^{2}$ and $1.53 \times 10^{-10} \mathrm{~m}^{2}$. The permeability is mainly a function of the porous media pore size (or particle size), and scales with the square of the latter. Using the values of the permeability above, it is found that the pore size would roughly lie between $9.5 \mu \mathrm{m}$ and $12.4 \mu \mathrm{m}$. The distribution of the size of the flocs making up the sludge samples used in our experiments may be rather stretched, and it is actually unknown. However the values found above are consistent with the values reported by the company which provided us with the sludge. This strongly supports our interpretation of the squeeze flow behavior of the sludge in terms of a Peclet number as defined above.

\section{Dewatering efficiency}

From the practical point of view, it is interesting to consider the amount of water released when the sludge is subjected to a squeeze deformation. We carried out tests in which the material has been squeezed out at different speeds. The sludge samples are weighed before and after the squeezing test. The mass fraction of the solid phase of the sample as a function of the squeeze speed is represented in Fig. 6. The mass of the solid phase is obtained by weighing the sample after drying for $24 \mathrm{~h}$ at $140{ }^{\circ} \mathrm{C}$. These results clearly show that the dewatering effect decreases when the squeeze speed increases. This is unexpected and may have important technological implications. These results are also consistent with the squeeze rheological analysis reported in the previous sub-section.

Figure 6 shows that the maximum solid concentration (or minimum water content) that can be obtained using squeeze procedure is about $52 \mathrm{wt} \%$ which is rather high.

\section{Conclusion}

We have investigated the rheological behavior of urban sewage sludges under two different flow conditions. When the sludge was subjected to small strain shear deformations, its behavior was solid-like and a yield

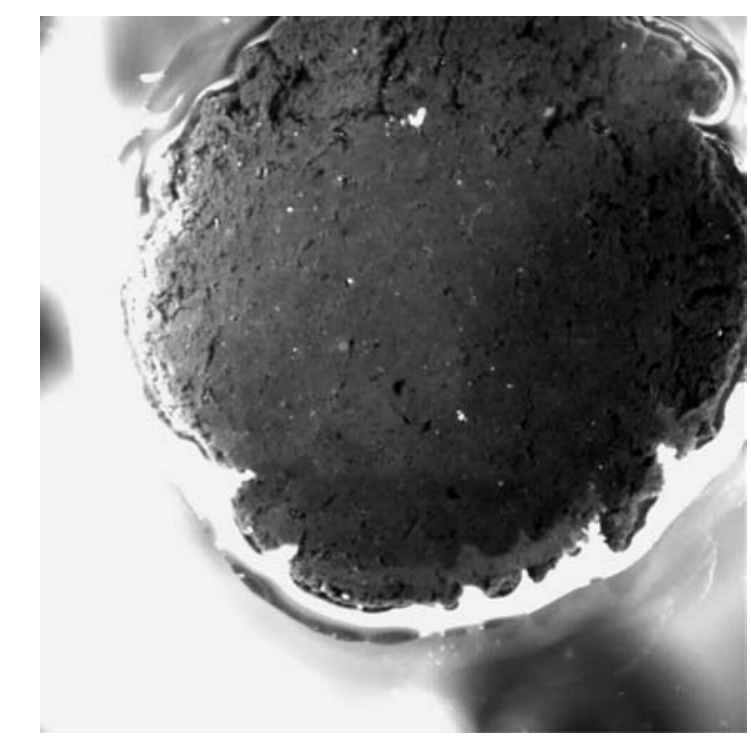

Fig. 6 At low speeds $(0.05 \mathrm{~mm} / \mathrm{s}$ here $)$ we can observe some liquid flow outside the material at the periphery of the sample

stress was measured. The second type of rheological tests considered was a squeeze test, which yielded much more complex and interesting results. Thus, similar to other paste-like materials already considered in the literature, we found that the squeeze deformation of the sludge resulted in two different mechanisms, depending upon the squeeze speed. For relatively large speeds, the sludge showed a shear-thinning power-law fluid behavior, and remained homogeneous during the deformation. For small speeds, both the rheological behavior and the microstructure of the sample were radically different: the squeeze force increased when the velocity was decreased and the material fractured. This behavior was attributed to a competition between the deformation of the sample as a whole and the filtration of the solvent through the porous media made up by the sludge flocs. Then, a Peclet number was defined to compare these two effects.

Finally, the dewatering efficiency as a function of the squeeze speed was considered. The results were consistent with the rheological study. It was found that the efficiency of the water extraction increases when the compression velocity decreases. This may have crucial consequences regarding the industrial sludge processing.

\section{References}

Adams MJ, Aydin I, Briscoe BJ, Sinha SK (1997) A finite element analysis of the squeeze flow of an elasto-viscoplastic paste material. J Non-Newtonian Fluid Mech 71(12):41-57
Barnes HA (1999) Yield stress - a review, or panta rei - everything flows? J NonNewtonian Fluid Mech 81(1/2):133-178
Baudez JC (2001) PhD thesis. Ecole Nationale du Génie Rural, des Eaux et des Forêts, Paris 
Baudez JC, Coussot P (2001) Rheology of aging, concentrated, polymeric suspensions: application to pasty sewage sludges. J Rheol 45:5

Campbell HW, Crescuollo PJ (1982) The use of rheology for sludge characterization. Water Sci Tech 14:475-489

Carman PC (1937) Trans Inst Chem Eng (London) 15:150-159

Chilton R, Stainsby R, Thompson S (1995) The design of sewage sludge pumping systems. J Hydraul Res 34:3

Delaye N, Poitou A, Chaouche M (2000) Squeeze flow of highly concentrated suspensions of spheres. J Non-Newtonian Fluid Mech 94(1):67-74

Honey HC, Pretorius WA (2000) Laminar flow pipe hydraulics of pseudo-plastic- thixotropic sewage sludges. Water SA 26(1):19-26

Kao N, Bhattacharya SN, Shanks RA, Coopes IH (1998) The effect of temperature on the viscoelastic properties of model and industrial dispersions. J Rheol 42(3):493-506

Lanteri B, Burlet H, Poitou A, Campion I (1996) Rheological behavior of a polymer-ceramic blend used for injection moulding. J Mater Sci 31:1751-1760

Legrand V (1997) PhD thesis. Universite Paris VI

Lotito V, Spinosa L, Mininni G, Antonacci R (1997) The rheology of sewage sludge at different steps of treatment. Water Sci Tech 36(11):79-85
Mulbarger MG, Copas SR, Kordic JR, Cash FM (1981) Pipeline friction losses for wastewater sludges. J Water Pollut Control Fed 53:1303-1313

Poitou A, Racineux G (2001) A squeezing experiment showing binder migration in concentrated suspensions. J Rheol 45:3

Racineux G (1999) PhD thesis. Ecole Normale Superieure de Cachan

Ravilly F (2000) PhD thesis. Institut National Polytechnique de Grenoble

Russel WB, Saville DA, Schowalter WR (1989) Colloidal dispersions. Cambridge University Press

Scott JR (1935) Trans Inst Rubber Ind 10:481-493 